BASIC RESEARCH

\title{
Irisin—behind the benefits of exercise
}

PGC-1 $\alpha$ in muscle induces the release into the circulation of a newly identified hormone, irisin, which exhibits regulatory effects on adipose tissue, a study in Nature reveals. This process increases energy expenditure and thermogenesis and leads to improvements in obesity and diabetes mellitus.

Exercise induces PGC-1 a expression in muscle, which is thought to mediate many of its health benefits. Transgenic mice that overexpress PGC-1 1 specifically in muscle are resistant to age-related obesity and diabetes mellitus, which suggested that PGC-1 a regulates muscle-secreted factors with effects on energy expenditure. However, the underlying mechanism remained elusive, and its elucidation was the aim of the study by Boström and co-workers.

Comparison of muscle gene-expression profiles of transgenic and wild-type mice revealed that PGC-1 $\alpha$ induced the expression of several genes with secreted protein products, including FNDC5. A new proteolytic cleavage product of this gene was identified and named irisin. Adenoviral expression of FNDC5, followed by analysis of gene expression in white adipose tissue, revealed that irisin induced a program of brown-fat-like development. This effect was associated with protection against diet-induced obesity and diabetes mellitus. Irisin was detected in murine and human plasma by Western blot analysis, demonstrating its release into the circulation, and its levels were increased by exercise, which suggests that it mediates exercise-induced 'browning' of white adipose tissue.

Given the antiobesity and antidiabetic effects of brown-fat formation in mice and the $100 \%$ homology between murine and human irisin, this hormone seems to show great therapeutic potential in these and related disorders.

\section{Andreia Cunha}

Original article Boström, P. et al. A PGC1- $a$-dependent myokine that drives brown-fat-like development of white fat and thermogenesis. Nature doi:10.1038/nature10777 